\title{
Clinical utility of targeted treatments in the management of epithelial ovarian cancer
}

\author{
This article was published in the following Dove Press journal: \\ Biologics:Targets and Therapy \\ 25 July 2012 \\ Number of times this article has been viewed
}

\author{
Cheryl Twu' \\ Ernest S Han² \\ 'Department of Women's Health, \\ Arrowhead Regional Medical Center, \\ Colton, CA, USA; ${ }^{2}$ Division of \\ Gynecologic Oncology, City of Hope, \\ Duarte, CA, USA
}

\begin{abstract}
Epithelial ovarian cancer is typically found in its advanced stages, where a combination of surgical debulking and platinum/taxane-based chemotherapy is recommended. Although over $70 \%-80 \%$ of patients achieve remission, a significant proportion develop recurrence of their disease. Additional cytotoxic chemotherapy, as well as surgery, is typically used to manage disease recurrence. Therapies that target specific pathways in cancer cells are rapidly developing in the laboratory and are increasingly being studied in patients with ovarian cancer. We review the current status of novel therapies in the management of epithelial ovarian cancer.

Keywords: angiogenesis, polyadenosine ribose pathway inhibitors, epidermal growth factor
\end{abstract} receptor, folate inhibitors

\section{Introduction}

In the United States, approximately 21,000 women are newly diagnosed with ovarian cancer each year. There are also 14,600 reported deaths from ovarian cancer each year, making it the fifth most common cause of death from cancer and the most common cause of death due to gynecologic cancer. ${ }^{1}$ Globally each year, there are approximately 200,000 new diagnoses of ovarian cancer and 125,000 deaths due to the disease. ${ }^{2,3}$ Mortality rates for ovarian cancer are high because patients often present with clinically advanced disease. Unfortunately, to date, we lack adequate screening tests for early detection of epithelial ovarian cancer (EOC).

Management of newly diagnosed EOC typically involves surgical exploration for histological confirmation, staging, and maximal cytoreductive effort if gross metastatic disease is present. The goal is to achieve complete resection or at least optimal tumor debulking, defined as residual tumor of less than $1 \mathrm{~cm}$ in diameter. ${ }^{4}$ Patients undergoing optimal tumor debulking have higher overall survival rates than those with suboptimal tumor debulking. ${ }^{5}$ For the majority of EOC patients, surgery is followed by chemotherapy with a combination of a regimen containing platinum and taxane, which leads to a $70 \%-80 \%$ complete response rate. ${ }^{6}$ Patients who have significant risks for surgery or are not surgical candidates for upfront debulking will often undergo neoadjuvant chemotherapy for 3-4 cycles and then proceed to interval debulking surgery followed by additional chemotherapy. In a Phase III trial by the European Gynecological Cancer group, this approach was shown to reduce mortality rates and adverse postoperative complications. ${ }^{7}$ However, the overall survival rate was considerably lower compared with other Phase III trials involving advanced EOC. ${ }^{7,8}$ Despite an excellent complete response rate after primary therapy, $70 \%-80 \%$ of patients will develop recurrence
Correspondence: Ernest S Han

City of Hope, Division of Gynecologic

Oncology, Duarte, CA 91010, USA

$\mathrm{Tel}+\mathrm{I} 6264717100$

Fax +16264717155

Email ehan@coh.org which permits unrestricted noncommercial use, provided the original work is properly cited. 
within a median of 18 months. ${ }^{8}$ The responsiveness of the cancer to subsequent treatment generally decreases and those that recur 6 months or less from initial treatment (platinumresistant disease) or have persistent disease despite primary upfront therapy have a poor prognosis. Clearly, novel approaches to the treatment of ovarian cancer are needed. Extensive research has led to improved understanding of the mechanisms involved in the pathogenesis of EOC. This has led to the rapid development of new agents that target pathways critical to ovarian cancer.

EOC has a unique pattern of metastases. Patients often have peritoneal disease that involves the surfaces of the bowel and other viscera within the intra-abdominal/pelvic cavity. Ovarian cancers are also heterogeneous in nature, making cure for these patients difficult. Multiple pathways in EOC have been determined to contribute to the pathogenesis and have recently been reviewed in detail. ${ }^{9}$ Table 1 lists key pathways of interest, where targeted agents have been developed. These pathways are important in driving ovarian cancer cell survival, proliferation, and metastasis. In addition, angiogenesis is thought to be critical in the development of ovarian cancer. This review will examine how the current clinical trials involving these pathways impact the management and treatment of patients with EOC.

\section{Targeted therapy in recurrent ovarian cancer}

We begin our discussion with patients having recurrent ovarian cancer, given that the development of new agents generally starts with women who have measurable recurrent disease. Despite a $70 \%-80 \%$ remission rate after upfront treatment of EOC, $70 \%-80 \%$ of patients develop recurrence and ultimately succumb to their disease. With numerous agents now available to treat patients with recurrent ovarian cancer, overall survival has steadily improved. However, clinicians/researchers continue to strive to find effective therapies while trying to minimize potential side effects to patients. Thus targeted therapies may become increasingly important to help maintain quality of life while still improving patient survival. Numerous Phase II trials have examined the effectiveness of various targeted therapies in both platinum-sensitive and platinum-resistant patients, and Phase III trials are increasingly incorporating quality of life measures.

\section{Angiogenesis inhibitors}

Angiogenesis has been an area of active research in EOC. Angiogenesis is the growth and development of new blood vessels, which is driven by vascular endothelial growth factor (VEGF). As tumors enlarge, they begin to outgrow their blood supply. Hypoxia develops, which in turn stimulates an increase in VEGF production by tumors. VEGF acts upon endothelial cells, which leads to complex changes and the increase of blood vessels. There is a family of receptors that has been identified on endothelial cells, and the major VEGF receptor involved in angiogenesis is VEGFR-2. VEGF receptors on tumor cells may also be critical to tumor growth. Angiogenesis involves a complex interaction between tumor cells, endothelial cells, and stromal cells.

Bevacizumab was one of the earliest angiogenesis inhibitors examined. Bevacizumab is a humanized monoclonal antibody directed against VEGF. Burger et al reported a Phase II Gynecologic Oncology Group study (GOG170D) which examined single-agent bevacizumab $(15 \mathrm{mg} / \mathrm{kg}$ intravenously every 3 weeks) in patients with recurrent EOC. ${ }^{10}$ A $21 \%$ response rate was noted and $40.3 \%$ had a 6 -month progression-free survival. However, another Phase II study of bevacizumab in patients with recurrent platinum-resistant EOC was stopped early due to increased rates of bowel perforation $(11.4 \%) .{ }^{11}$ Despite this, patients experienced a $16 \%$ response rate, and the median progression-free survival duration was 4.4 months. These studies indicated that

Table I Key pathways involved in the pathogenesis of epithelial ovarian cancer

\begin{tabular}{ll}
\hline Pathway & Function \\
\hline Angiogenesis & $\begin{array}{l}\text { Formation of new blood vessels to support cancer growth and metastasis; involved in vascular } \\
\text { permeability and vasodilation. } \\
\text { Epidermal growth factor receptor }\end{array}$ \\
$\begin{array}{l}\text { Membrane receptors that dimerize and activate tyrosine kinase activity, leading to activation of MAPK } \\
\text { and PI3K/AKT pathways. This leads to increased cell growth and decreased apoptosis. }\end{array}$ \\
Folate pathway & $\begin{array}{l}\text { Esential for purine biosynthesis; folate transporter overexpression increases folate to cancer cells, } \\
\text { Polyadenosine diphosphate-ribose }\end{array}$ \\
Polymerase & $\begin{array}{l}\text { leads to increased single-strand breaks, and thus DNA double-strand breaks. BRCA mutations lead to } \\
\text { defective double-strand break repairs, resulting in chromosome instability and cell death. }\end{array}$ \\
PI3K-AKT-mTOR pathway & Regulates apoptosis, metabolism, cell proliferation, angiogenesis, and cell growth.
\end{tabular}

Abbreviations: PI3K, phosphoinositide 3-kinase; mTOR, mammalian target of rapamycin; MAPK, mitogen activated protein kinase. 
bevacizumab itself was active in the treatment of recurrent EOC, which was unique from other cancer types where bevacizumab activity was only noted in combination with other cytotoxic agents.

Bevacizumab has also been studied in combination with other agents. Garcia et al reported on the combination of bevacizumab and cytoxan administered in a metronomic fashion and found significant clinical activity (24\% response rate, $56 \%$ progression-free survival at 6 months). However, the combination of bevacizumab and erlotinib, an epidermal growth factor receptor (EGFR) tyrosine kinase inhibitor, failed to demonstrate improved clinical activity (15\% overall response rate). ${ }^{12}$

The first and only Phase III placebo-controlled study of bevacizumab in patients with recurrent ovarian cancer was recently completed, ie, OCEANS (study of carboplatin and gemcitabine plus bevacizumab in patients with ovary, peritoneal, or fallopian tube carcinoma). This study was reported at the 2011 American Society of Clinical Oncology meeting. ${ }^{13}$ The results show a significant improvement in median progression-free survival in the bevacizumab arm (12.4 months) compared with the placebo arm (8.4 months). Currently the Gynecologic Oncology Group is studying carboplatin and paclitaxel combined with placebo versus bevacizumab in patients with platinum-sensitive first-line recurrent disease (GOG213). Patients are initially determined if they are appropriate surgical candidates, and if so, are randomized to secondary cytoreduction or to no surgery. Patients who are not surgical candidates or have completed the surgical arm of the trial are then randomized to either carboplatin-paclitaxel or carboplatin-paclitaxel plus bevacizumab followed by maintenance bevacizumab.

Given the significant serious toxicities (eg, bowel perforation, fistula formation, thrombosis) noted in the trials, further studies are needed to improve the selection of patients who will respond to therapy and avoid unnecessary exposure and risk to bevacizumab. It is also interesting that there may be a rebound effect of worsening survival upon discontinuing bevacizumab treatments based on recent laboratory findings. ${ }^{14,15}$ Further studies are needed to determine whether continuing bevacizumab or other antiangiogenic agents should be considered in patients.

Although bevacizumab has been the most studied antiangiogenesis agent, numerous other angiogenesis inhibitors have also been examined and are mostly in Phase II trials. Aflibercept (or VEGF Trap) is a protein that contains portions of the VEGFR-1 and VEGFR-2 extracellular domains that are fused to the Fc portion of IgG. Aflibercept binds
VEGFA and VEGFB and placental growth factor. Tew et al reported on the preliminary results of a randomized Phase II trial of aflibercept at the 2007 American Society of Clinical Oncology meeting. ${ }^{16}$ Patients with heavily pretreated recurrent ovarian cancer were randomized to receive either $2 \mathrm{mg} / \mathrm{kg}$ or $4 \mathrm{mg} / \mathrm{kg}$ dosing. Five partial responses (11\%) were noted, along with significant grade 3-4 toxicities, including hypertension, proteinuria, encephalopathy, and renal failure. Of note, there were two bowel perforations reported. Aflibercept has also been used to treat symptomatic malignant ascites in patients with recurrent ovarian cancer. ${ }^{17}$ The mean time to repeat paracentesis was longer when patients were treated with aflibercept ( 55 days) as compared with placebo (22 days). However, three fatal gastrointestinal perforations were noted in patients treated with aflibercept.

The small-molecule tyrosine kinase inhibitors that target the VEGF receptor have also been examined. Sorafenib is a kinase inhibitor that targets the VEGF receptor and also targets Raf, platelet-derived growth factor (PDGF) receptor, Flt3, and c-kit. In a Phase II study by the Gynecologic Oncology Group, patients with recurrent ovarian cancer treated with sorafenib showed only a $3.4 \%$ partial response, while $34 \%$ had stable disease and $24 \%$ were progression-free at 6 months. ${ }^{18}$ Sorafenib has also been combined with cytotoxic agents (gemcitabine) and the results have been similarly poor ( $4.7 \%$ objective response rate). ${ }^{19}$ Another multitargeted kinase inhibitor, sunitinib, has also been examined, and only one of 30 patients demonstrated a partial response $(3.3 \%)$, while $53 \%$ had stable disease. ${ }^{20}$ Although the results have not been more encouraging, additional studies are needed to determine whether novel combinations of these agents with either cytotoxic or other targeted agents can improve patient outcomes.

Cediranib is a potent VEGF inhibitor with activity against VEGFR-1, VEGFR-2, and VEGFR-3, and has shown some promise in initial studies. A Phase II study using singleagent oral cediranib in recurrent EOC showed a $17 \%$ partial response rate, with stable disease noted in $13 \%$. Median progression-free survival was 5.2 months. ${ }^{21}$ Cediranib is currently being tested in the International Collaboration for Ovarian Neoplasia (ICON) trial, in which patients are randomized to three arms, ie, platinum-based chemotherapy with placebo maintenance, platinum-based chemotherapy plus cediranib with placebo maintenance, or platinum-based chemotherapy plus cediranib with cediranib maintenance. Cediranib so far has shown no significant toxicities in this double-blind study, allowing expansion into the second phase of this trial. ${ }^{22}$ 
Additional drugs have been developed to novel targets in the angiogenesis pathway and are in early clinical development (Table 2). These include AMG 386, which targets the angiopoietin pathway and leads to inhibition of tumor endothelial cell proliferation and growth, ${ }^{23}$ and BIBF 1120 , which is a triple angiokinase inhibitor that blocks the VEGF, PDGF, and fibroblast growth factor receptors. ${ }^{24}$ In contrast with blocking the formation of new vessels, drugs that target the existing tumor vasculature (vascular disrupting agents) are in early research (Table 2).

\section{Epidermal growth factor receptor}

The EGFR is part of a family of tyrosine kinase receptors known as ErbB. This family also includes ErbB-2 (HER-2), ErbB-3, and ErbB-4. Ligand binding to these receptors leads to homodimerization and heterodimerization, which in turn leads to activation of the signal transduction pathway and ultimately promotes cell proliferation, angiogenesis, metastasis, and inhibition of apoptosis. ${ }^{25}$ EGFR has been found to be overexpressed in 35\%-70\% of ovarian cancers. ${ }^{25}$ Numerous EGFR inhibitors have been developed and tested in patients with ovarian cancer. These inhibitors include small molecules that block the tyrosine kinase activity of the receptor and monoclonal antibodies that target the extracellular domain.

Erlotinib and gefitinib, both small-molecule inhibitors, have been examined in patients with recurrent ovarian cancer. Single-agent Phase II studies of both drugs have been disappointing, with poor response rates noted $(6 \%$ for erlotinib $^{26} ; 0 \%-4 \%$ for gefitinib $\left.{ }^{27,28}\right)$. Even when patients had detectable levels of target EGFR and phospho-EGFR, which were decreased with gefitinib therapy, there was no clinically associated benefit. ${ }^{29}$ Phase II studies combining erlotinib or gefitinib with cytotoxic chemotherapies did demonstrate significant clinical response. Patients with recurrent ovarian cancer and up to two prior lines of chemotherapy who were treated with erlotinib and carboplatin demonstrated a 57\% overall response rate in platinum-sensitive disease, while a 7\% overall response rate was noted in platinum-resistant disease. ${ }^{30}$ A Phase II study of gefitinib with paclitaxel and carboplatin as second-line treatment for ovarian cancer demonstrated a $62 \%$ overall response rate in platinum-sensitive patients compared with a $19.2 \%$ overall response rate in platinum-resistant patients. ${ }^{31}$ However, the extent of contribution of the EGFR inhibitor in these combinations is unclear.

Monoclonal antibodies directed towards EGFR have been developed. Cetuximab, a chimeric monoclonal antibody that blocks the binding of epidermal growth factor to its receptor, was examined in a Phase II study of patients with persistent or recurrent ovarian cancer. However, there was minimal activity noted, with only one of 25 patients noted to have a partial response and nine having stable disease. ${ }^{32}$ In a Gynecologic Oncology Group Phase II study, when cetuximab was combined with carboplatin in patients who were EGFR-positive, nine of 28 patients had an objective response, with eight noted to have stable disease. ${ }^{33}$ Despite this, further evaluation of this combination in a second stage of accrual was halted due to the response rate not meeting the study criteria. ${ }^{33}$ EMD72000 (matuzumab), a humanized anti-EGFR monoclonal antibody, was also tested in a heavily pretreated group of patients with platinum-resistant ovarian cancer. No responses were noted in this Phase II trial. ${ }^{34}$

Similarly poor response rates have been also noted for anti-HER 2 antibodies. Patients with recurrent ovarian cancer treated with trastuzumab had an overall response rate of $7.3 \%$. Interestingly, only $11.4 \%$ of patients screened for this study demonstrated $2+/ 3+$ immunohistochemical levels of HER2, which were required for treatment. Another antibody, pertuzumab, that blocks HER2 dimerization, showed only a $4.3 \%$ response rate. ${ }^{35}$ When combined with gemcitabine in platinum-resistant ovarian cancer, a randomized Phase II study noted that the overall response rate and hazard ratio were improved compared with the placebo arm (overall response rate 13.8 versus 4.6; progression-free survival hazards ratio 0.32). ${ }^{36}$ Current studies involve panitumumab, a fully human monoclonal antibody to EGFR (Table 2).

\section{Other tyrosine kinase inhibitors}

PDGF and KIT represent novel targets in ovarian cancers. Henriksen et al found that PDGF and its receptor PDGFR- $\alpha$, are present in $73 \%$ and $36 \%$ of malignant ovarian tumors, respectively. ${ }^{37}$ This may lead to autocrine stimulation of ovarian cancer cells. ${ }^{38}$ Recently, PDGF-B has been shown to be important in recruiting pericytes to the endothelium. ${ }^{39}$ Pericytes help to stabilize and maintain function of the microvasculature. Preclinical data suggest that inhibition of pericyte function may improve antiangiogenic therapies. ${ }^{40,41}$ KIT is a transmembrane glycoprotein and a member of the tyrosine kinase family. ${ }^{42}$ Found in numerous malignancies, KIT is activated by its ligand, stem cell factor, and also by mutations in the c-kit gene, both of which lead to downstream activation of signaling pathways. ${ }^{42}$

Thus far, inhibitors of the PDGF and KIT pathways have been disappointing. Sunitinib, a multikinase inhibitor, the targets of which include VEGF receptors 1-3 and PDGF $\alpha$ 
Table 2 Summary of current clinical trials of targeted therapies for recurrent ovarian cancer*

\begin{tabular}{|c|c|c|c|c|}
\hline Class & Drug & Intervention & Phase & Protocol ID \\
\hline \multirow{33}{*}{$\begin{array}{l}\text { Angiogenesis } \\
\text { inhibitors }\end{array}$} & Bevacizumab & Paclitaxel-topotecan- & III & MO22224, 2009-0II400-33, \\
\hline & & doxorubicin & & NCT009769II \\
\hline & & $\begin{array}{l}\text { Paclitaxel-topotecan- } \\
\text { doxorubicin, bevacizumab }\end{array}$ & & \\
\hline & Bevacizumab & Carboplatin, paclitaxel, & III & MO22923, 2010-019525-34, \\
\hline & & bevacizumab & & NCTOI 239732 \\
\hline & Bevacizumab & Carboplatin, bevacizumab & II & 2008-000878-20, S-20080033, \\
\hline & & & & 26।2-3754, NCT007447|8 \\
\hline & Bevacizumab & Bevacizumab, gemcitabine & II & AVF43I4S, NCT0II3I039 \\
\hline & Bevacizumab, sorafenib & Bevacizumab, sorafenib & II & 070058, 07-C-0058, NCl-07-C-0058, \\
\hline & (VEGF inhibitor) & & & NCI-P6840, 7358, NCT004362I5 \\
\hline & Sorafenib & Sorafenib and topotecan & II & TRIAS 2009, NCT0I04789I \\
\hline & AMG 386 & Paclitaxel plus AMG 386 & III & TRINOVAI: 20090508 \\
\hline & & Paclitaxel plus placebo & & NCT0I 204749 \\
\hline & AMG 386 & Doxorubicin or topotecan & I & 20070I82, NCT00770536 \\
\hline & & and AMG 386 & & \\
\hline & BIBF II 20 & Carboplatin, doxorubicin, & I & II99.119, 2010-022523-30, \\
\hline & & BIBF II 20 in dose escalation & & NCT0I3I4I05 \\
\hline & BIBF II 20 & Doxorubicin, BIBF II 20 & I, II & GYNI0-I49, NCT0I 485874 \\
\hline & & in dose escalation & & \\
\hline & BIBF 1120 & Carboplatin, doxorubicin, & 1 & I I990I I7, NCT0I329549 \\
\hline & & BIBF II 20 & & \\
\hline & Pazopanib & Paclitaxel, pazopanib & II & GOG-0I86J, NCT0I468909 \\
\hline & & Paclitaxel, placebo & & \\
\hline & Pazopanib & Pazopanib & II & GEICO-I002, 20I0-020439-38, \\
\hline & & & & NCTOI2620I4 \\
\hline & Pazopanib & Doxorubicin, pazopanib & I, II & SCRI GYN 26, NCTOI035658 \\
\hline & & in dose escalation & & \\
\hline & Pazopanib & Pazopanib and cyclophosphamide & I, II & 3107000, NCT0I 238770 \\
\hline & Pazopanib & Carboplatin, paclitaxel, & I, II & CDR0000703686, EORTC-55092, \\
\hline & & pazopanib in dose escalation & & EU-2III9, EUDRACT-2010-024077-39, \\
\hline & & & & NCT0I40227I \\
\hline & Cediranib Olaparib & Cediranib and olaparib & I, II & DFCI 09-293, U0ICA062490-I6S2, \\
\hline & & Olaparib only & & NCI 8348, NCT0III 6648 \\
\hline \multirow{6}{*}{$\begin{array}{l}\text { Vascular } \\
\text { disrupting agents }\end{array}$} & Ombrabulin & Ombrabulin & II & EFCI0260, 2010-02463I-16, \\
\hline & & Placebo & & UIIII-III8-5437, NCT0I332656 \\
\hline & Fosbretabulin & Bevacizumab, fosbretabulin & ॥ & GOG-0I86I, NCT0I3052I3 \\
\hline & & Bevacizumab alone & & \\
\hline & EP-I00 & IEP-I00, paclitaxel & II & ACTI260I, UIIII-II24-2062, \\
\hline & & Paclitaxel alone & & NCT0I485848 \\
\hline \multirow[t]{12}{*}{ PARP inhibitors } & AZD228I & Carboplatin & 1 & 080092, 08-C-0092, NCT0I4454I8 \\
\hline & & AZD228I in dose escalation & & \\
\hline & Veliparib & $\begin{array}{l}\text { Carboplatin, doxorubicin, } \\
\text { veliparib in dose escalation }\end{array}$ & 1 & GOG-9927, NCT0I459380 \\
\hline & Veliparib & Doxorubicin, veliparib & I & CDR00006749I7, AECM-000248, \\
\hline & & in dose escalation & & 00-0248, 8475, NCT0II 45430 \\
\hline & Veliparib (ABT-888) & $A B T-888$ in dose escalation & I & UPCI 08-I2I, 8282, NCT00892736, \\
\hline & & & & CINJ-0508I0 \\
\hline & Veliparib (ABT-888) & Cyclophosphamide, ABT-888 & II & II 0080, II-C-0080, NCT0I306032 \\
\hline & Veliparib & Veliparib & I, II & Veli-BRCA, NCT0I472783 \\
\hline & Veliparib (ABT-888) & Topotecan and ABT-888 & I, II & MAYO-MC086I, MC086I, 8329, \\
\hline & & in dose escalation & & NCTOI0I28I7 \\
\hline & Iniparib (BSI-20I) & $\begin{array}{l}\text { Carboplatin, gemcitabine, } \\
\text { iniparib }\end{array}$ & II & TCDII504, 20090208, NCT0I033292 \\
\hline $\begin{array}{l}\mathrm{PI} 3 \mathrm{~K} / \mathrm{AKT} / \mathrm{mTOR} \\
\text { inhibitors }\end{array}$ & MK-2206 & MK-2206 & II & I0-402, NCTOI 283035 \\
\hline
\end{tabular}


Table 2 (Continued)

\begin{tabular}{|c|c|c|c|c|}
\hline Class & Drug & Intervention & Phase & Protocol ID \\
\hline & MM-I2I & Paclitaxel, MM-I2I & II & MM-I 2 I-04-02-08 (ARDI I586), \\
\hline & & Paclitaxel alone & & NCT0I447706 \\
\hline & Temsirolimus & Temsirolimus and bevacizumab & II & $\begin{array}{l}\text { MAYO-MC0845, MC0845, } 8233 \text {, } \\
\text { NCTOI0I0I } 26\end{array}$ \\
\hline & Everolimus & $\begin{array}{l}\text { Carboplatin, doxorubicin, } \\
\text { everolimus in dose escalation }\end{array}$ & 1 & $\begin{array}{l}\text { IRB I0-0I9, NCl-20II-00055, } \\
\text { NCT0I28I5I } 4\end{array}$ \\
\hline & Everolimus RAD00I & RAD00I and bevacizumab & II & 09-0I-RAD00IBEV, NCT0I03I38I \\
\hline & Everolimus & Bevacizumab & II & GOG-0I86G, NCT0088669I \\
\hline & & Everolimus, bevacizumab & & \\
\hline \multirow[t]{4}{*}{$\begin{array}{l}\text { Folate pathway } \\
\text { inhibitors }\end{array}$} & Pralatrexate & $\begin{array}{l}\text { Carboplatin and pralatrexate } \\
\text { in dose escalation }\end{array}$ & I, II & I0-II3, NCTOII88876 \\
\hline & Pemetrexed & Pemetrexed and carboplatin & II & MMC-08-04-097, NCT0I00I9I0 \\
\hline & Farletuzumab & Carboplatin, taxane, farletuzumab & III & MORAb003-004, NCT00849667 \\
\hline & MORAb-003 & Carboplatin, taxane only & & \\
\hline \multirow[t]{3}{*}{ EGFR inhibitor } & Panitumumab & $\begin{array}{l}\text { Carboplatin, doxorubicin, } \\
\text { panitumumab }\end{array}$ & II & $\begin{array}{l}\text { GMIHO-008/2009_AG56, } \\
2010-018849-59, \text { AG56, }\end{array}$ \\
\hline & & Carboplatin, doxorubicin & & NCT0I38862I \\
\hline & Panitumumab & Panitumumab and gemcitabine & II & WIH 20050782, NCTOI 296035 \\
\hline
\end{tabular}

Note: *Data for table obtained from http://www.cancer.gov/clinicaltrials and was accessed on February 9, 2012.

Abbreviations: VEGF, vascular endothelial growth factor; PARP, polyadenosine ribose pathway.

and $\beta$, was shown to have modest activity in patients with recurrent ovarian cancer (3.3\% partial response and 53\% stable disease).$^{20}$ Imatinib is a tyrosine kinase inhibitor that targets KIT and PDGF receptor $\alpha$ and $\beta$. Like the smallmolecule EGFR inhibitors, single-agent imatinib has minimal activity alone, with an overall response rate of $0 \%-2 \% .^{27,43-45}$ Combination therapy of imatinib with cytotoxic chemotherapy did not demonstrate a striking improvement in response. Imatinib was combined with docetaxel in heavily pretreated platinum-resistant ovarian cancer patients, and a $21.7 \%$ overall response rate was noted. ${ }^{46}$ Imatinib with weekly paclitaxel yielded a $25 \%$ overall response rate. ${ }^{47}$

\section{Polyadenosine ribose pathway inhibitors}

The polyadenosine ribose pathway (PARP) pathway is an exciting new target that is gaining momentum in clinical trials. The concept of targeting PARP has been recently reviewed by Javle and Curtin. ${ }^{48}$ PARP is a nuclear enzyme that functions to repair DNA damage (specifically singlestrand breaks) in cells. Inhibition of PARP leads to more single-strand breaks. Persistent single-strand breaks lead to collapse of the replication fork, which then leads to replication-associated double-strand DNA breaks. Typically, double-strand breaks are repaired by homologous recombination, which is mediated in part by BRCA1 and BRCA2 proteins. Mutations in BRCA1 and BRCA2 occur in about $10 \%$ of all EOC patients, and predispose women to increased risk of breast and ovarian cancer. More recently, inactivation of BRCA1 may be a common event in the pathogenesis of sporadic cases of EOC. This is thought to occur via various mechanisms, such as loss of heterozygosity, hypermethylation, and haploinsufficiency. ${ }^{49}$

Importantly, when PARP is inhibited and the homologous recombination pathway is defective (eg, due to BRCA1 or 2 mutation), this leads to increased double-strand breaks which are lethal if they persist or lead to genomic instability due to utilization of alternative, error-prone repair pathways.

Several studies involving PARP inhibitors have been recently completed in ovarian cancer patients. Fong et al reported a Phase I trial which involved patients with BRCA1 or BRCA2 mutations, with $35 \%$ of patients having ovarian cancer. Olaparib (AZD2281) was examined and found to be generally well tolerated..$^{50}$ The cohort of BRCA-mutated patients with ovarian, fallopian tube, and primary peritoneal cancers were expanded and examined for response to olaparib. ${ }^{51}$ Twenty of 50 patients had a complete or partial response. Despite decreased response to olaparib in platinum-refractory or platinum-resistant disease, a significant response to treatment was still noted, with a $41.7 \%$ response rate in platinum-resistant disease by RECIST (Response Evaluation Criteria In Solid Tumors) criteria. There were no responders in the platinumrefractory group by RECIST criteria, but a $15.4 \%$ response rate was noted by Gynecologic Cancer Intergroup criteria, which utilizes CA125 for determination of response. Treatments were well tolerated, with mild gastrointestinal symptoms and fatigue being the most common toxicities reported.

Olaparib was examined in a Phase II study involving women with BRCA1 or BRCA2 mutations and recurrent 
ovarian cancers. ${ }^{52}$ Audeh et al examined two cohorts which involved two different dosing schedules for olaparib (cohort 1 , $400 \mathrm{mg}$ twice daily; cohort 2, $100 \mathrm{mg}$ twice daily), with objective tumor response by RECIST criteria being the primary endpoint of the study. A $33 \%$ and $13 \%$ response rate was noted for cohort 1 and 2, respectively. Again, the treatments were well tolerated, with nausea and fatigue being the most common adverse events reported. There were two deaths reported in cohort 1 , one from intestinal perforation and disease progression, and the other due to development of acute myeloid leukemia. Studies of other PARP inhibitors (AZD2281, BSI-201, ABT-888) are currently ongoing (see Table 2).

\section{Folate inhibitors}

Targeting of the folate pathways has been utilized in the past for other types of cancers, such as leukemias. As our understanding of the pathway has evolved, new inhibitors have been generated. Two such inhibitors, pemetrexed and farletuzumab, have been examined in the clinical setting. Pemetrexed is a multitargeting antifolate drug that inhibits multiple DNA synthesis pathway enzymes and gains access into the cell by a reduced folate carrier. ${ }^{53}$ Folate receptor alpha, which transports folate across the cell membrane, has been found to be upregulated in ovarian cancer cells. ${ }^{54,55}$ A humanized monoclonal antibody, farletuzumab, has been recently developed and binds to folate receptor alpha. ${ }^{56}$

There are two Phase II studies that have reported on singleagent pemetrexed in patients with platinum-resistant ovarian cancer, and significant activity was reported in both trials. ${ }^{57-58}$ Miller et al noted a $21 \%$ response rate, with $35 \%$ of patients having stable disease. ${ }^{57}$ Vergote et al compared two different doses of pemetrexed $\left(500 \mathrm{mg} / \mathrm{m}^{2}\right.$ versus $\left.900 \mathrm{mg} / \mathrm{m}^{2}\right) .{ }^{58}$ The response rate was about the same (9\%-10\%) at either dose. In addition, progression-free survival and overall survival were essentially similar. Bone marrow toxicities were similarly noted. Pemetrexed has also been combined with carboplatin in platinum-sensitive recurrent ovarian cancers. The overall response rate was $51.1 \%$ with $31.1 \%$ with stable disease..$^{59}$ The combination was generally well tolerated. Recently, farletuzumab has been examined in a Phase I study. ${ }^{60}$ It was well tolerated and appears acceptable to advance to Phase II studies. Current studies involving inhibition of the folate pathway are noted in Table 2.

\section{Other targeted agents in recurrent ovarian cancer}

The mammalian target of rapamycin (mTOR) is a serine/ threonine protein kinase involved in the regulation of cell growth. mTOR is a downstream target of the AKT/PI3K signaling pathway. Inhibitors have been developed against mTOR and are showing activity in ovarian cancer.

Enzastaurin is an oral protein kinase $\mathrm{C}$ inhibitor (specifically protein kinase $\mathrm{C}$ beta) and an inhibitor of the PI3K/AKT signaling pathway. A Phase II study by the Gynecologic Oncology Group in patients with recurrent ovarian or primary peritoneal cancers treated with enzastaurin showed an $11 \%$ progression-free survival of $\geq 6$ months, with a $7 \%$ partial response rate noted. ${ }^{61}$ There appeared to be low activity noted. However, the treatment was well tolerated, with no grade 4 adverse events reported.

Recently, Behbakht et al reported a Phase II trial of the mTOR inhibitor, temsirolimus. There was clinically significant activity noted, with $24 \%$ of patients describing $\mathrm{a} \geq 6$-month progression-free survival. ${ }^{62}$ However, the study did not meet Gynecologic Oncology Group criteria to continue studying the drug in a Phase III trial. Additional mTOR inhibitors are currently undergoing investigation (everolimus, MK-2206, MM-121, Table 2). MK-2206 is an allosteric AKT inhibitor that has been found in preclinical studies to enhance the effectiveness of cytotoxic chemotherapies. ${ }^{63} \mathrm{MM}-121$ is an antibody directed against ErbB3, which activates PI3K signaling in EGFR. ${ }^{64}$ Both of these novel PI3K/AKT pathway inhibitors are currently under investigation (Table 2).

\section{Management of newly diagnosed EOC}

Current standard chemotherapy includes treatment with intravenous carboplatin and paclitaxel every three weeks. Several studies have tried to alter this regimen slightly by either changing the mode of administration of the drug or changing the dose rate. One study performed by the Japanese Gynecologic Oncology Group changed the dose of paclitaxel given over one hour from $180 \mathrm{mg} / \mathrm{m}^{2}$ intravenously every 3 weeks to a dose-dense regimen of $80 \mathrm{mg} / \mathrm{m}^{2}$ intravenously once a week. ${ }^{65}$ Both groups received a mean of six cycles of treatment. ${ }^{65}$ The main toxicity was hematologic, causing a delay in or discontinuation of treatment, especially in the dose-dense group. The main adverse event was neutropenia, which was found in both the dose-dense and conventional dose groups. The study found a significantly longer progression-free survival and higher rate of overall survival at 3 years in patients treated with the dose-dense regimen. ${ }^{65}$ The Japanese group is also working on a dose-dense protocol using intraperitoneal chemotherapy in which they propose to demonstrate that intraperitoneal chemotherapy is superior to conventional intravenous administration. ${ }^{66}$ 
Other trials have already demonstrated benefit from changing the route of administration of chemotherapy from intravenous to intraperitoneal. The Phase III trial led by Armstrong for the Gynecologic Oncology Group showed a significant increase in median progression-free survival by 5 months for patients who received intraperitoneal cisplatin and paclitaxel compared with those who received intravenous chemotherapy after optimal debulking. In addition, median overall survival was increased by approximately 15 months in the intraperitoneal group, with a $25 \%$ decrease in risk of death. Although significantly more patients in the intraperitoneal group experienced serious adverse events, including gastrointestinal, pulmonary, cardiovascular or renal events, most of these did not lead to discontinuation of treatment. Quality of life was also similar between the groups after one year of treatment. ${ }^{67}$

Other studies have tried to improve upon standard chemotherapy for ovarian cancer by addition of a third chemotherapeutic drug to the regimen. A large multicenter, multiarm trial proposed by the Gynecologic Oncology Group in conjunction with the ICON group (GOG182-ICON5) launched in 2001, compared the addition of gemcitabine, methoxypolyethylene glycosylated liposomal doxorubicin, or topotecan to carboplatin and paclitaxel. ${ }^{68}$ To date, the study has not shown a significant improvement in progression-free survival or overall survival in patients to suggest a change from standard intravenous carboplatin and paclitaxel. ${ }^{68}$

\section{Targeted agents in front-line therapy for EOC}

Angiogenesis inhibitors, in particular, bevacizumab, are one of the most well studied target agents in cancer treatment. The formation of new vasculature is a key component to the spread and proliferation of cancer. Angiogenesis may be mediated along several different pathways, including VEGF, PDGF, EGFR, and tyrosine kinase inhibitors. Promising studies have added bevacizumab, a VEGF inhibitor, to standard treatment regimens with carboplatin and paclitaxel.

One study by the Gynecologic Oncology Group (GOG218) randomized patients with advanced EOC after debulking surgery into one of three treatment groups: standard paclitaxel, carboplatin, and placebo; paclitaxel, carboplatin and bevacizumab added on cycle 2 with placebo maintenance; or paclitaxel, carboplatin, and bevacizumab, followed by 15 months of maintenance bevacizumab. ${ }^{69}$ Arm 3 showed an improvement of 4 months in progressionfree survival compared with arm 1 , and demonstrated that the addition of bevacizumab showed benefit over conventional chemotherapy. Although the results may be statistically different, their clinical relevance may be tempered by the potential high costs related to bevacizumab therapy. ${ }^{70}$ In addition, there was no significant difference in overall survival between the three arms, but it should be noted that the study was not powered for overall survival. ${ }^{69}$

Another Phase III trial has also supported the use of bevacizumab in the upfront setting. A Phase III trial by the Gynecologic Cancer Intergroup (ICON7) randomized patients with stage II-IV EOC to carboplatin, paclitaxel and placebo, or to carboplatin, paclitaxel, and bevacizumab, with additional maintenance bevacizumab after primary therapy. ${ }^{71}$ This study found a 1.5-month improvement in progressionfree survival in patients treated with bevacizumab compared with the control arm (hazard ratio for progression or death $0.81 ; 95 \%$ confidence interval: $0.70-0.94 ; P=0.004)$. Overall survival data were not mature at the time of reporting.

In both GOG218 and ICON7, bevacizumab was well tolerated and the rates of bowel perforation were not statistically different between the controls and bevacizumab-containing arms. It was interesting to note that the progression-free survival curves for both studies seem to converge after bevacizumab was discontinued and suggests that prolonged bevacizumab maintenance therapy may benefit patients. Further studies are needed to address this finding. An additional question that remains is whether the routes of administration of chemotherapy (ie, intravenous versus intraperitoneal) matter. Studies already show the benefits of the intraperitoneal route, though its toxicity remains higher than with conventional intravenous administration. Some researchers have analyzed the cost-effectiveness of intraperitoneal chemotherapy because of potential longer-term hospital stays for serious toxicity. ${ }^{72}$ Others argue that if overall survival is increased with intraperitoneal administration, then it may prove to be a good value. ${ }^{73}$ A recently closed Gynecologic Oncology Group study (GOG-252) was designed to compare intravenous carboplatin with intraperitoneal carboplatin and cisplatin along with the addition of bevacizumab. In the first phase of the study, patients are randomized into three treatment arms: weekly intravenous paclitaxel + intravenous carboplatin every 3 weeks + intravenous bevacizumab starting cycle 2 ; weekly intravenous paclitaxel + intraperitoneal carboplatin + intravenous bevacizumab starting cycle 2 ; or intravenous paclitaxel cycle $1+$ intraperitoneal cisplatin + intraperitoneal paclitaxel cycle $2+$ intravenous bevacizumab starting cycle 2 . The second phase of the trial will maintain all three groups on intravenous bevacizumab starting on cycle $7 .{ }^{74}$ Other studies involving bevacizumab as well as 
other antiangiogenesis agents in first-line therapy for EOC are noted in Table 3.

Few other targeted agents have been designed as initial therapy for advanced EOC, primary peritoneal cancer, and fallopian tube cancer. In a Phase II trial by Konner et al, cetuximab, a monoclonal antibody synthetically designed to block tyrosine kinase inhibitors, was added to paclitaxel and carboplatin, and patients were then continued on weekly cetuximab for 6 months. ${ }^{75}$ However, while treatment was generally well tolerated, cetuximab had only limited efficacy compared with conventional chemotherapy. ${ }^{75}$ Another Phase II trial led by Vasey et al added erlotinib, a tyrosine kinase inhibitor, to docetaxel and carboplatin. ${ }^{76}$ The overall response rate was $52 \%$, with five of 24 patients having a complete response for at least 6 months. The results are encouraging and future studies are necessary. Current first-line therapy with PARP inhibitors and PI3K/mTOR inhibitors are ongoing (Table 3 ).

\section{Maintenance therapy for primary ovarian caner}

Once a complete response to treatment with cytoreductive surgery and chemotherapy has been established, a variety of maintenance therapies are used to prevent relapse or recurrence. A multicenter Phase III trial by the Southwest Oncology Group randomized patients to receive three cycles or 12 cycles of maintenance intravenous paclitaxel after a complete response to conventional platinum-based and paclitaxel-based treatment. ${ }^{77} \mathrm{~A}$ follow-up to their initial results shows a median progression-free survival of
22 months for 12 cycles of paclitaxel and 14 months for those who received only three cycles. The overall survival rate was better by 5 months (53 versus 48 months) in the 12-cycle group, but the results were not statistically significant. ${ }^{78}$

In contrast, another study by the Italian Gynecologic Oncology Group, looked at consolidation treatment with intravenous paclitaxel for six cycles versus observation in patients who had a complete response to first-line standard chemotherapy for advanced ovarian cancer. Two hundred patients were enrolled, and a median follow-up duration of 43 months showed no significant improvement in progressionfree survival or overall survival between the two arms. ${ }^{79}$ Similar negative findings were also noted in EOC patients with stage IA/B (grade 3 or clear cell), IC, or II disease. ${ }^{80}$ In this study, patients were randomized to observation or to maintenance paclitaxel $40 \mathrm{mg} / \mathrm{m}^{2} /$ week $\times 24$ weeks, and patients in the maintenance arm had a higher incidence of peripheral neuropathy, infection/fever, and dermatologic events. ${ }^{80}$ In a study by Berek et al, patients were randomized to receive either oregovomab, a monoclonal antibody to CA125, or placebo after front-line carboplatin and paclitaxel treatment for advanced EOC. ${ }^{81}$ This Phase III study unfortunately did not show any advantage of oregovomab over placebo. ${ }^{81}$

An ongoing protocol by the Gynecologic Oncology Group (GOG 212) is studying the novel compound, CT-2103 (Xyotax $\left.{ }^{\mathrm{TM}}\right)$, a synthetic taxane derived from conjugation of paclitaxel with a polyglutamate polymer. Preliminary data show reduced toxicity with this novel compound compared with paclitaxel. Like paclitaxel, CT-2103 does not demonstrate

Table 3 Summary of current clinical trials of first-line targeted therapies for ovarian cancer*

\begin{tabular}{|c|c|c|c|c|}
\hline Class & Drug & Intervention & Phase & Protocol ID \\
\hline \multirow[t]{6}{*}{$\begin{array}{l}\text { Angiogenesis } \\
\text { inhibitors }\end{array}$} & Bevacizumab & Carboplatin, bevacizumab, paclitaxel in dose escalation & 1 & $\begin{array}{l}\text { 2010C0049, OSU 09149, } \\
\text { NCT0I219777 }\end{array}$ \\
\hline & Bevacizumab & $\begin{array}{l}\text { IP carboplatin, IV paclitaxel, IV bevacizumab } \\
\text { in dose escalation }\end{array}$ & I & $\begin{array}{l}\text { 2010CO062, OSU09115, } \\
\text { NCT } 01220154\end{array}$ \\
\hline & Bevacizumab & $\begin{array}{l}\text { Standard chemotherapy plus bevacizumab for } 30 \text { months } \\
\text { Standard chemotherapy plus bevacizumab for } 15 \text { months }\end{array}$ & III & $\begin{array}{l}\text { AGO-OVAR I7, } \\
\text { NCTOI } 462890\end{array}$ \\
\hline & Bevacizumab & Carboplatin, paclitaxel, bevacizumab & II & 2009-0I86, NCT0I097746 \\
\hline & AMG 386 & Carboplatin, paclitaxel, AMG 386 & III & TRINOVA3: 2010II29/ \\
\hline & & Carboplatin, paclitaxel, placebo & & ENGOT-ov2, NCT0I493505 \\
\hline \multirow[t]{2}{*}{ PARP inhibitors } & Veliparib & $\begin{array}{l}\text { IV carboplatin, paclitaxel, bevacizumab, veliparib } \\
\text { IP cisplatin } \\
\text { IV/IP paclitaxel, bevacizumab, veliparib }\end{array}$ & I & GOG-9923, NCT0098965I \\
\hline & ABT-767 & ABT-767 dose escalation study & 1 & $\begin{array}{l}\text { MI0-976, 20I0-020795-37, } \\
\text { NCT0I339650 }\end{array}$ \\
\hline \multirow{2}{*}{$\begin{array}{l}\mathrm{PI} 3 \mathrm{~K} / \mathrm{mTOR} \\
\text { inhibitor }\end{array}$} & BKMI20 & BKMI 20 once daily & II & II-2II, NCT0I50I604 \\
\hline & Temsirolimus & Carboplatin, paclitaxel, temsirolimus $\geq$ temsirolimus consolidation & II & GOG-0268, NCT0II96429 \\
\hline
\end{tabular}

Note: *Data for table obtained from http://www.cancer.gov/clinicaltrials and was accessed February 9, 2012.

Abbreviations: IV, intravenous; IP, intraperitoneal; PARP, polyadenosine ribose pathway; PI3K, phosphoinositide 3-kinase; mTOR, mammalian target of rapamycin. 
an increase in response rate with an increase in dosage. A $135 \mathrm{mg} / \mathrm{m}^{2}$ dose of CT-2103 appears to be as safe and as efficacious as unconjugated paclitaxel at $135 \mathrm{mg} / \mathrm{m}^{2}{ }^{82}$ Another ongoing multicenter, international Phase III study involves the use of pazopanib, a VEGF receptor and PDGF receptor inhibitor, as monotherapy for consolidation treatment following complete response to first-line chemotherapy. ${ }^{83}$ This study has finished data accrual and is set for primary data results by 2012 .

\section{Conclusion}

Advances in therapies are changing the way we manage patients with EOC. Through the testing of novel agents in patients with recurrent disease, changes to first-line therapy are occurring. The addition of bevacizumab to a platinum/taxane-based regimen may become the new standard if the mature overall survival data support continued benefit in patients. However, the debate over utilizing intravenous versus intraperitoneal chemotherapy is not resolved. It is assumed that use of bevacizumab in either treatment setting will be beneficial, but there are no specific clinical trials addressing the use of novel therapeutic agents in patients undergoing intraperitoneal chemotherapy.

The use of targeted agents in patients with recurrent disease is constantly evolving. Because many of these new drugs seem to show fewer side effects compared with cytotoxic chemotherapy, it will be interesting to see how these new therapies will be incorporated into the management of patients with EOC. Should we be utilizing targeted agents much sooner in the patient's therapy? Should we always combine the targeted agents with cytotoxic therapies? Certainly clinical trials will be important to help address these issues. However, improved biomarkers are desperately needed to determine the efficacy and response of targeted agents and to aid in determining whether the targeted agent requires further clinical evaluation or should be abandoned. Cost issues and access to these new agents will also need to be addressed as new targeted agents come onto the market.

\section{Acknowledgment}

We are grateful to Yesenia Donahue for her editorial assistance in preparing this paper for publication.

\section{Disclosure}

The authors report no conflicts of interest in this work.

\section{References}

1. Jemal A, Siegel R, Xu J, Ward E. Cancer statistics, 2010. CA Cancer J Clin. 2010;60(5):277-300.

2. Parkin DM, Bray F, Ferlay J, Pisani P. Global cancer statistics, 2002. CA Cancer J Clin. 2005;55(2):74-108.
3. Sankaranarayanan R, Ferlay J. Worldwide burden of gynaecological cancer: the size of the problem. Best Pract Res Clin Obstet Gynaecol. 2006;20(2):207-225.

4. Wakabayashi MT, Lin PS, Hakim AA. The role of cytoreductive/ debulking surgery in ovarian cancer. J Natl Compr Canc Netw. 2008;6(8):803-810.

5. Bristow RE, Tomacruz RS, Armstrong DK, Trimble EL, Montz FJ. Survival effect of maximal cytoreductive surgery for advanced ovarian carcinoma during the platinum era: a meta-analysis. J Clin Oncol. 2002;20(5):1248-1259.

6. Ozols RF, Bundy BN, Greer BE, et al. Phase III trial of carboplatin and paclitaxel compared with cisplatin and paclitaxel in patients with optimally resected stage III ovarian cancer: a Gynecologic Oncology Group study. J Clin Oncol. 2003;21(17):3194-3200.

7. Vergote I, Trope CG, Amant F, et al. Neoadjuvant chemotherapy or primary surgery in stage IIIC or IV ovarian cancer. $N$ Engl J Med. 2010;363(10):943-953.

8. DiSaia PJ, Creasman WT. Epithelial ovarian cancer. In: Clinical Gynecologic Oncology. 7th ed. St Louis, MO: Mosby Elsevier; 2007.

9. Yap TA, Carden CP, Kaye SB. Beyond chemotherapy: targeted therapies in ovarian cancer. Nat Rev Cancer. 2009;9(3):167-181.

10. Burger RA, Sill MW, Monk BJ, Greer BE, Sorosky JI. Phase II trial of bevacizumab in persistent or recurrent epithelial ovarian cancer or primary peritoneal cancer: a Gynecologic Oncology Group Study. J Clin Oncol. 2007;25(33):5165-5171.

11. Cannistra SA, Matulonis UA, Penson RT, et al. Phase II study of bevacizumab in patients with platinum-resistant ovarian cancer or peritoneal serous cancer. J Clin Oncol. 2007;25(33):5180-5186.

12. Nimeiri HS, Oza AM, Morgan RJ, et al. Efficacy and safety of bevacizumab plus erlotinib for patients with recurrent ovarian, primary peritoneal, and fallopian tube cancer: a trial of the Chicago, $\mathrm{PMH}$, and California Phase II Consortia. Gynecol Oncol. 2008;110(1):49-55.

13. Aghajanian C, Finkler NJ, Rutherford T, et al. OCEANS: A randomized, double-blinded, placebo-controlled Phase III trial of chemotherapy with or without bevacizumab (BEV) in patients with platinum-sensitive recurrent epithelial ovarian (EOC), primary peritoneal (PPC), or fallopian tube cancer (FTC). J Clin Oncol. 2011;29 Suppl:Abstr LBA5007.

14. Ebos JM, Lee CR, Cruz-Munoz W, Bjarnason GA, Christensen JG, Kerbel RS. Accelerated metastasis after short-term treatment with a potent inhibitor of tumor angiogenesis. Cancer Cell. 2009;15(3): 232-239.

15. Paez-Ribes M, Allen E, Hudock J, et al. Antiangiogenic therapy elicits malignant progression of tumors to increased local invasion and distant metastasis. Cancer Cell. 2009;15(3):220-231.

16. Tew WP, Colombo N, Ray-Coquard I, et al. VEGF-Trap for patients (pts) with recurrent platinum-resistant epithelial ovarian cancer (EOC): Preliminary results of a randomized, multicenter Phase II study. J Clin Oncol. 2007;25(18S):5508.

17. Gotlieb WH, Amant F, Advani S, et al. Intravenous aflibercept for treatment of recurrent symptomatic malignant ascites in patients with advanced ovarian cancer: a Phase 2, randomised, double-blind, placebocontrolled study. Lancet Oncol. 2012;13(2):154-162.

18. Matei D, Sill MW, Lankes HA, et al. Activity of sorafenib in recurrent ovarian cancer and primary peritoneal carcinomatosis: a Gynecologic Oncology Group trial. J Clin Oncol. 2011;29(1):69-75.

19. Welch SA, Hirte HW, Elit L, et al. Sorafenib in combination with gemcitabine in recurrent epithelial ovarian cancer: a study of the Princess Margaret Hospital Phase II Consortium. Int J Gynecol Cancer. 2010;20(5):787-793.

20. Biagi JJ, Oza AM, Chalchal HI, et al. A Phase II study of sunitinib in patients with recurrent epithelial ovarian and primary peritoneal carcinoma: an NCIC Clinical Trials Group Study. Ann Oncol. 2011;22(2):335-340.

21. Matulonis UA, Berlin S, Ivy P, et al. Cediranib, an oral inhibitor of vascular endothelial growth factor receptor kinases, is an active drug in recurrent epithelial ovarian, fallopian tube, and peritoneal cancer. J Clin Oncol. 2009;27(33):5601-5606. 
22. Raja FA, Griffin CL, Qian W, et al. Initial toxicity assessment of ICON6: a randomised trial of cediranib plus chemotherapy in platinum-sensitive relapsed ovarian cancer. Br J Cancer. 2011;105(7):884-889.

23. Herbst RS, Hong D, Chap L, et al. Safety, pharmacokinetics, and antitumor activity of AMG 386, a selective angiopoietin inhibitor, in adult patients with advanced solid tumors. J Clin Oncol. 2009;27(21):3557-3565.

24. Hilberg F, Roth GJ, Krssak M, et al. BIBF 1120: triple angiokinase inhibitor with sustained receptor blockade and good antitumor efficacy. Cancer Res. 2008;68(12):4774-4782.

25. Marshall J. Clinical implications of the mechanism of epidermal growth factor receptor inhibitors. Cancer. 2006;107(6):1207-1218.

26. Gordon AN, Finkler N, Edwards RP, et al. Efficacy and safety of erlotinib $\mathrm{HCl}$, an epidermal growth factor receptor (HER1/EGFR) tyrosine kinase inhibitor, in patients with advanced ovarian carcinoma: results from a Phase II multicenter study. Int J Gynecol Cancer. 2005;15(5):785-792.

27. Posadas EM, Kwitkowski V, Kotz HL, et al. A prospective analysis of imatinib-induced c-KIT modulation in ovarian cancer: a Phase II clinical study with proteomic profiling. Cancer. 2007;110(2):309-317.

28. Schilder RJ, Sill MW, Chen X, et al. Phase II study of gefitinib in patients with relapsed or persistent ovarian or primary peritoneal carcinoma and evaluation of epidermal growth factor receptor mutations and immunohistochemical expression: a Gynecologic Oncology Group study. Clin Cancer Res. 2005;11(15):5539-5548.

29. Posadas EM, Liel MS, Kwitkowski V, et al. A Phase II and pharmacodynamic study of gefitinib in patients with refractory or recurrent epithelial ovarian cancer. Cancer. 2007;109(7):1323-1330.

30. Hirte H, Oza A, Swenerton K, et al. A Phase II study of erlotinib (OSI774) given in combination with carboplatin in patients with recurrent epithelial ovarian cancer (NCIC CTG IND.149). Gynecol Oncol. 2010;118(3):308-312.

31. Pautier P, Joly F, Kerbrat P, et al. Phase II study of gefitinib in combination with paclitaxel $(\mathrm{P})$ and carboplatin $(\mathrm{C})$ as second-line therapy for ovarian, tubal or peritoneal adenocarcinoma (1839IL/0074). Gynecol Oncol. 2010;116(2):157-162.

32. Schilder RJ, Pathak HB, Lokshin AE, et al. Phase II trial of single agent cetuximab in patients with persistent or recurrent epithelial ovarian or primary peritoneal carcinoma with the potential for dose escalation to rash. Gynecol Oncol. 2009;113(1):21-27.

33. Secord AA, Blessing JA, Armstrong DK, et al. Phase II trial of cetuximab and carboplatin in relapsed platinum-sensitive ovarian cancer and evaluation of epidermal growth factor receptor expression: a Gynecologic Oncology Group study. Gynecol Oncol. 2008;108(3):493-499.

34. Seiden MV, Burris HA, Matulonis U, et al. A Phase II trial of EMD72000 (matuzumab), a humanized anti-EGFR monoclonal antibody, in patients with platinum-resistant ovarian and primary peritoneal malignancies. Gynecol Oncol. 2007;104(3):727-731.

35. Gordon MS, Matei D, Aghajanian C, et al. Clinical activity of pertuzumab (rhuMAb 2C4), a HER dimerization inhibitor, in advanced ovarian cancer: potential predictive relationship with tumor HER2 activation status. J Clin Oncol. 2006;24(26):4324-4332.

36. Makhija S, Amler LC, Glenn D, et al. Clinical activity of gemcitabine plus pertuzumab in platinum-resistant ovarian cancer, fallopian tube cancer, or primary peritoneal cancer. J Clin Oncol. 2010;28(7):1215-1223.

37. Henriksen R, Funa K, Wilander E, Backstrom T, Ridderheim M, Oberg K. Expression and prognostic significance of platelet-derived growth factor and its receptors in epithelial ovarian neoplasms. Cancer Res. 1993;53(19):4550-4554.

38. Matei D, Emerson RE, Lai YC, et al. Autocrine activation of PDGFRalpha promotes the progression of ovarian cancer. Oncogene. 2006;25(14):2060-2069.

39. Raza A, Franklin MJ, Dudek AZ. Pericytes and vessel maturation during tumor angiogenesis and metastasis. Am J Hematol. 2010;85(8):593-598
40. Lu C, Kamat AA, Lin YG, et al. Dual targeting of endothelial cells and pericytes in antivascular therapy for ovarian carcinoma. Clin Cancer Res. 2007;13(14):4209-4217.

41. Lu C, Shahzad MM, Moreno-Smith M, et al. Targeting pericytes with a PDGF-B aptamer in human ovarian carcinoma models. Cancer Biol Ther. 2010;9(3):176-182.

42. Heinrich MC, Blanke CD, Druker BJ, Corless CL. Inhibition of KIT tyrosine kinase activity: a novel molecular approach to the treatment of KIT-positive malignancies. J Clin Oncol. 2002;20(6):1692-1703.

43. Alberts DS, Liu PY, Wilczynski SP, et al. Phase II trial of imatinib mesylate in recurrent, biomarker positive, ovarian cancer (Southwest Oncology Group Protocol S0211). Int J Gynecol Cancer. 2007;17(4):784-788.

44. Coleman RL, Broaddus RR, Bodurka DC, et al. Phase II trial of imatinib mesylate in patients with recurrent platinum- and taxane-resistant epithelial ovarian and primary peritoneal cancers. Gynecol Oncol. 2006;101(1):126-131.

45. Schilder RJ, Sill MW, Lee RB, et al. Phase II evaluation of imatinib mesylate in the treatment of recurrent or persistent epithelial ovarian or primary peritoneal carcinoma: a Gynecologic Oncology Group study. J Clin Oncol. 2008;26(20):3418-3425.

46. Matei D, Emerson RE, Schilder J, et al. Imatinib mesylate in combination with docetaxel for the treatment of patients with advanced, platinumresistant ovarian cancer and primary peritoneal carcinomatosis: a Hoosier Oncology Group trial. Cancer. 2008;113(4):723-732.

47. Safra T, Andreopoulou E, Levinson B, et al. Weekly paclitaxel with intermittent imatinib mesylate (Gleevec): tolerance and activity in recurrent epithelial ovarian cancer. Anticancer Res. 2010;30(9): 3243-3247.

48. Javle M, Curtin NJ. The role of PARP in DNA repair and its therapeutic exploitation. Br J Cancer. 2011;105(8):1114-1122.

49. Weberpals JI, Clark-Knowles KV, Vanderhyden BC. Sporadic epithelial ovarian cancer: clinical relevance of BRCA1 inhibition in the DNA damage and repair pathway. J Clin Oncol. 2008;26(19):3259-3267.

50. Fong PC, Boss DS, Yap TA, et al. Inhibition of poly(ADP-ribose) polymerase in tumors from BRCA mutation carriers. $N$ Engl J Med. 2009;361(2):123-134.

51. Fong PC, Yap TA, Boss DS, et al. Poly(ADP)-ribose polymerase inhibition: frequent durable responses in BRCA carrier ovarian cancer correlating with platinum-free interval. J Clin Oncol. 2010;28(15):2512-2519.

52. Audeh MW, Carmichael J, Penson RT, et al. Oral poly(ADP-ribose) polymerase inhibitor olaparib in patients with BRCA1 or BRCA2 mutations and recurrent ovarian cancer: a proof-of-concept trial. Lancet. 2010;376(9737):245-251.

53. Adjei AA. Pemetrexed: a multitargeted antifolate agent with promising activity in solid tumors. Ann Oncol. 2000;11(10):1335-1341.

54. Kalli KR, Oberg AL, Keeney GL, et al. Folate receptor alpha as a tumor target in epithelial ovarian cancer. Gynecol Oncol. 2008;108(3):619-626.

55. Markert S, Lassmann S, Gabriel B, et al. Alpha-folate receptor expression in epithelial ovarian carcinoma and non-neoplastic ovarian tissue. Anticancer Res. 2008;28(6A):3567-3572.

56. Ebel W, Routhier EL, Foley B, et al. Preclinical evaluation of MORAb-003, a humanized monoclonal antibody antagonizing folate receptor-alpha. Cancer Immun. 2007; 7:6.

57. Miller DS, Blessing JA, Krasner CN, et al. Phase II evaluation of pemetrexed in the treatment of recurrent or persistent platinum-resistant ovarian or primary peritoneal carcinoma: a study of the Gynecologic Oncology Group. J Clin Oncol. 2009;27(16):2686-2691.

58. Vergote I, Calvert H, Kania M, Kaiser C, Zimmermann AH, Sehouli J. A randomised, double-blind, Phase II study of two doses of pemetrexed in the treatment of platinum-resistant, epithelial ovarian or primary peritoneal cancer. Eur J Cancer. 2009;45(8):1415-1423.

59. Matulonis UA, Horowitz NS, Campos SM, et al. Phase II study of carboplatin and pemetrexed for the treatment of platinum-sensitive recurrent ovarian cancer. J Clin Oncol. 2008;26(35):5761-5766. 
60. Konner JA, Bell-McGuinn KM, Sabbatini P, et al. Farletuzumab, a humanized monoclonal antibody against folate receptor alpha, in epithelial ovarian cancer: a Phase I study. Clin Cancer Res. 2010;16(21):5288-5295.

61. Usha L, Sill MW, Darcy KM, et al. A Gynecologic Oncology Group Phase II trial of the protein kinase C-beta inhibitor, enzastaurin and evaluation of markers with potential predictive and prognostic value in persistent or recurrent epithelial ovarian and primary peritoneal malignancies. Gynecol Oncol. 2011;121(3):455-461.

62. Behbakht K, Sill MW, Darcy KM, et al. Phase II trial of the mTOR inhibitor, temsirolimus and evaluation of circulating tumor cells and tumor biomarkers in persistent and recurrent epithelial ovarian and primary peritoneal malignancies: a Gynecologic Oncology Group study. Gynecol Oncol. 2011;123(1):19-26.

63. Hirai H, Sootome H, Nakatsuru Y, et al. MK-2206, an allosteric Akt inhibitor, enhances antitumor efficacy by standard chemotherapeutic agents or molecular targeted drugs in vitro and in vivo. Mol Cancer Ther. 2010;9(7):1956-1967.

64. Schoeberl B, Faber AC, Li D, et al. An ErbB3 antibody, MM-121, is active in cancers with ligand-dependent activation. Cancer Res. 2010;70(6):2485-2494.

65. Katsumata N, Yasuda M, Takahashi F, et al. Dose-dense paclitaxel once a week in combination with carboplatin every 3 weeks for advanced ovarian cancer: a Phase 3, open-label, randomised controlled trial. Lancet. 2009;374(9698):1331-1338.

66. Fujiwara K, Aotani E, Hamano T, et al. A randomized Phase II/III trial of 3 weekly intraperitoneal versus intravenous carboplatin in combination with intravenous weekly dose-dense paclitaxel for newly diagnosed ovarian, fallopian tube and primary peritoneal cancer. Jpn J Clin Oncol. 2011;41(2):278-282.

67. Armstrong DK, Bundy B, Wenzel L, et al. Intraperitoneal cisplatin and paclitaxel in ovarian cancer. $N$ Engl J Med. 2006;354(1):34-43.

68. Bookman MA, Brady MF, McGuire WP, et al. Evaluation of new platinum-based treatment regimens in advanced-stage ovarian cancer: a Phase III Trial of the Gynecologic Cancer Intergroup. J Clin Oncol. 2009;27(9):1419-1425.

69. Burger RA, Brady MF, Bookman MA, et al. Incorporation of bevacizumab in the primary treatment of ovarian cancer. $N$ Engl J Med. 2011;365(26):2473-2483

70. Cohn DE, Kim KH, Resnick KE, O’Malley DM, Straughn JM Jr. At what cost does a potential survival advantage of bevacizumab make sense for the primary treatment of ovarian cancer? A cost-effectiveness analysis. J Clin Oncol. 2011;29(10):1247-1251.

71. Perren TJ, Swart AM, Pfisterer J, et al. A Phase 3 trial of bevacizumab in ovarian cancer. $N$ Engl J Med. 2011;365(26):2484-2496.

72. Bristow RE, Santillan A, Salani R, et al. Intraperitoneal cisplatin and paclitaxel versus intravenous carboplatin and paclitaxel chemotherapy for Stage III ovarian cancer: a cost-effectiveness analysis. Gynecol Oncol. 2007;106(3):476-481.
73. Havrilesky LJ, Secord AA, Darcy KM, Armstrong DK, Kulasingam S. Cost effectiveness of intraperitoneal compared with intravenous chemotherapy for women with optimally resected stage III ovarian cancer: a Gynecologic Oncology Group study. J Clin Oncol. 2008;26(25):4144-4150.

74. Walker JL. Intraperitoneal chemotherapy for ovarian cancer: 2009 goals. Gynecol Oncol. 2009;112(3):439-440.

75. Konner J, Schilder RJ, DeRosa FA, et al. A Phase II study of cetux$\mathrm{imab} /$ paclitaxel/carboplatin for the initial treatment of advanced-stage ovarian, primary peritoneal, or fallopian tube cancer. Gynecol Oncol. 2008;110(2):140-145.

76. Vasey PA, Gore M, Wilson R, et al. A Phase Ib trial of docetaxel, carboplatin and erlotinib in ovarian, fallopian tube and primary peritoneal cancers. Br J Cancer. 2008;98(11):1774-1780.

77. Markman M, Liu PY, Wilczynski S, et al. Phase III randomized trial of 12 versus 3 months of maintenance paclitaxel in patients with advanced ovarian cancer after complete response to platinum and paclitaxel-based chemotherapy: a Southwest Oncology Group and Gynecologic Oncology Group trial. J Clin Oncol. 2003;21(13):2460-2465.

78. Markman M, Liu PY, Moon J, et al. Impact on survival of 12 versus 3 monthly cycles of paclitaxel $\left(175 \mathrm{mg} / \mathrm{m}^{2}\right)$ administered to patients with advanced ovarian cancer who attained a complete response to primary platinum-paclitaxel: follow-up of a Southwest Oncology Group and Gynecologic Oncology Group Phase 3 trial. Gynecol Oncol. 2009;114(2):195-198.

79. Pecorelli S, Favalli G, Gadducci A, et al. Phase III trial of observation versus six courses of paclitaxel in patients with advanced epithelial ovarian cancer in complete response after six courses of paclitaxel/ platinum-based chemotherapy: final results of the After-6 protocol 1 . J Clin Oncol. 2009;27(28):4642-4648.

80. Mannel RS, Brady MF, Kohn EC, et al. A randomized Phase III trial of IV carboplatin and paclitaxel x 3 courses followed by observation versus weekly maintenance low-dose paclitaxel in patients with early-stage ovarian carcinoma: a Gynecologic Oncology Group Study. Gynecol Oncol. 2011;122(1):89-94.

81. Berek J, Taylor P, McGuire W, Smith LM, Schultes B, Nicodemus CF. Oregovomab maintenance monoimmunotherapy does not improve outcomes in advanced ovarian cancer. J Clin Oncol. 2009;27(3): 418-425.

82. Sabbatini P, Sill MW, O’Malley D, Adler L, Secord AA. A Phase II trial of paclitaxel poliglumex in recurrent or persistent ovarian or primary peritoneal cancer (EOC): a Gynecologic Oncology Group Study. Gynecol Oncol. 2008;111(3):455-460.

83. GlaxoSmithKline. Efficacy and safety of pazopanib monotherapy after first line chemotherapy in ovarian, fallopian tube, or primary peritoneal cancer, 2009. Available from: http:/www.clinicaltrials.gov/ct2/show/ NCT00866697. Accessed April 4, 2012.

\section{Publish your work in this journal}

Biologics: Targets \& Therapy is an international, peer-reviewed journal focusing on the patho-physiological rationale for and clinical application of Biologic agents in the management of autoimmune diseases, cancers or other pathologies where a molecular target can be identified. This journal is indexed on PubMed Central, CAS, EMBase, Scopus
Dovepress

and the Elsevier Bibliographic databases. The manuscript management system is completely online and includes a very quick and fair peerreview system, which is all easy to use. Visit http://www.dovepress com/testimonials.php to read real quotes from published authors. 